\title{
Disease severity and prophylactic measures in patients with cutaneous lupus erythematosus: results of a worldwide questionnaire-based study
}

\author{
Dominik Samotij ${ }^{1,2}$, Justyna Szczęch ${ }^{1}$, Victoria P. Werth ${ }^{3,4}$, Fukumi Furukawa ${ }^{5}$, Annegret Kuhn ${ }^{6}$, Jacek C. Szepietowski², \\ Adam Reich ${ }^{1}$
}

${ }^{1}$ Department of Dermatology, University of Rzeszow, Rzeszow, Poland

${ }^{2}$ Department of Dermatology, Venereology and Allergology, Wroclaw Medical University, Wroclaw, Poland

${ }^{3}$ Corporal Michael J. Crescenz Veterans Affairs Medical Center, Philadelphia, PA, USA

${ }^{4}$ Department of Dermatology, Perelman School of Medicine at the University of Pennsylvania, Philadelphia, PA, USA

${ }^{5}$ Department of Dermatology, Wakayama Medical University, Wakayama, Japan

${ }^{6}$ University Hospital Muenster, Muenster, Germany

Adv Dermatol Allergol 2018; XXXV (2): 192-198

DOI: https://doi.org/10.5114/ada.2018.75242

\begin{abstract}
Introduction: Due to a wide array of dermatologic manifestations, assessment of disease severity in cutaneous lupus erythematosus (CLE) remains challenging. Given a need for some standardization in this field, we conducted a worldwide questionnaire-based study among physicians experienced in CLE management.

Aim: We asked about CLE assessment, their prophylactic measures advised to patients, and treatment recommendations.

Material and methods: A total of 83 completed questionnaires were received. Participating physicians recommended assessing disease severity at each patient's visit (39.1\%), monthly (4.9\%) or at least every third month (17.3\%). Almost half of the responding physicians (47.0\%) waited $2-3$ months before identifying a specific treatment option as not effective.

Results: The vast part of the participants informed their patients about of the risks of sun exposure and advised adequate preventive measures. Smoking was less frequently a matter of discussion between physicians and their patients. Recommendations for the timing of CLE severity assessment likely depends on disease severity and the type of therapeutic intervention.

Conclusions: Proper patient education about effective prophylactic measures should be included during routine CLE patient consultations.
\end{abstract}

Key words: cutaneous lupus erythematosus, chronic cutaneous lupus erythematosus, discoid lupus erythematosus, lupus erythematosus tumidus, subacute cutaneous lupus erythematosus, prophylaxis.

\section{Introduction}

Skin involvement is seen in about $70-85 \%$ of all patients with lupus erythematosus (LE) and represents the first sign of the disease in about one quarter of patients [1]. The treatment of cutaneous manifestations of LE still remains a challenge, as therapeutic options currently available for cutaneous LE (CLE) are limited and medications are prescribed based on the personal experience of physicians and often lack proper randomized control trial testing which would justify their "off-label" use [2]. The efficacy and safety of many conventionally applied therapies have mainly been evaluated in a few open- label studies and retrospective analyses. However, the challenge includes both a lack of randomized controlled studies assessing treatment efficacy and inconsistent outcome measures and trial design for patients with CLE. At least sixty instruments measure SLE activity, with the British Isles Lupus Assessment Group (BILAG) and Systemic Lupus Erythematosus Disease Activity Index (SLEDAI) most frequently used. However, only three of these SLE instruments are suitable for assessing the skin involvement of CLE [3]. The only validated tool specifically used for evaluation of disease activity and damage in CLE patients is the Cutaneous Lupus Erythematosus

Address for correspondence: Adam Reich MD, PhD, Department of Dermatology, University of Rzeszow, 2 Szopena St, 35 -55 Rzeszow, Poland, phone: +48 605 076722, e-mail: adamandrzejreich@gmail.com Received: 28.12.2016, accepted: 29.01.2017. 
Disease Area and Severity Index (CLASI) [4, 5]. The CLASI is responsive in numerous SLE and CLE trials, and has been used to monitor improvement of the skin in SLE and CLE patients in large international multicenter trials and in prospective assessments of CLE patients undergoing standard of care treatments [6-8]. Assessment of skin in routine care can use either the CLASI, which takes 5 min to perform but does require training, or a global physicians assessment by visual analogue scale, which is less time-consuming, but also more subjective. Time points for assessments of the skin depends on the severity of skin involvement, and during studies depends on the mechanism of action of an intervention. Thus, total standardization for specific time points is difficult.

\section{Aim}

In order to have a better insight into physician habits regarding their evaluation of CLE patients in the clinical setting and to understand how frequently treatment efficacy is assessed, we performed a worldwide questionnaire-based study among physicians with a special interest in CLE in order to better characterize the frequency and methods of evaluating CLE severity in different centers. In addition, we asked all participants about their routine recommendations concerning prophylactic measures for their CLE patients.

\section{Material and methods}

This was a worldwide questionnaire-based study. The questionnaire was sent via email to each center dealing with CLE patients, as well as to participants of the $3^{\text {rd }}$ International Conference on Cutaneous Lupus Erythematosus (ICCLE) held in Edinburgh (Scotland) in May 2013.

A total of 83 physicians from Japan ( $n=41,49.4 \%)$, European countries ( $n=23,27.7 \%)$, the USA $(n=15$, $18.1 \%)$, Canada $(n=2,2.4 \%)$ and the Republic of South Africa $(n=2,2.4 \%)$ with expertise in dealing with patients with CLE completed the questionnaire containing questions about treatment recommendations, patient's assessment and prophylactic measures they advise to patients. Data on treatment recommendations were published elsewhere [9].

There were 78 (94.0\%) dermatologists, 3 (3.6\%) dermatologists/rheumatologists, and 2 (2.4\%) rheumatologists. Their age was $48.1 \pm 9.9$ years (median: 47 years), with a range of 33-68 years. The male : female ratio was $2: 1$. Most of participants (88.0\%) worked at university hospitals. The chronic cutaneous lupus erythematosus (CCLE) subtype predominated among the patients treated by participating physicians, followed by subacute cutaneous lupus erythematosus (SCLE) and acute cutaneous lupus erythematosus (ACLE). Detailed data on participants are demonstrated in Table 1.

\section{Statistical analysis}

All results were analyzed using the software package Statistica 12.0 (Statsoft, Krakow, Poland). Descriptive statistics included frequencies, median, minimal and maximal values. The significance of the observed differences between groups has been determined by Mann-Whitney $U$ test, and $\chi^{2}$ test with Yates correction, if necessary. A $p$-value lower than 0.05 was considered as statistically significant.

\section{Results}

\section{Assessment of CLE patients}

Among the participating physicians, the treatment efficacy most often was assessed according to the Physician Global Assessment (PGA) of disease severity ( $n=$ $58,69.9 \%$ ) or based on the patient's opinion regarding the efficacy of the applied therapy $(n=33,39.8 \%)$. Other methods, like CLASI $(n=26,31.3 \%)$, structured evaluation of patient's quality of life (QoL) $(n=12,14.5 \%)$, or

Table 1. Characteristics of participating physicians.

\begin{tabular}{|c|c|}
\hline Parameter & Value \\
\hline \multicolumn{2}{|l|}{ Age [years]: } \\
\hline Median & 47 \\
\hline Range & $33-68$ \\
\hline \multicolumn{2}{|l|}{ Gender: } \\
\hline Male & $55(66.3 \%)$ \\
\hline Female & $28(33.7 \%)$ \\
\hline \multicolumn{2}{|l|}{ Main place of work: } \\
\hline University hospital & $73(88.0 \%)$ \\
\hline Hospital, other & $9(10.8 \%)$ \\
\hline Private outpatient clinic & $1(1.2 \%)$ \\
\hline \multicolumn{2}{|c|}{ Duration of professional experience [years]: } \\
\hline Median & 20 \\
\hline Range & $5-40$ \\
\hline \multicolumn{2}{|c|}{ Average number of CLE patients seen per month: } \\
\hline 0-1 patient & $6(7.2 \%)$ \\
\hline 2-5 patients & $29(34.9 \%)$ \\
\hline $6-10$ patients & 19 (22.9\%) \\
\hline 11-20 patients & $13(15.7 \%)$ \\
\hline$>20$ patients & $13(15.7 \%)$ \\
\hline No data & $3(3.6 \%)$ \\
\hline \multicolumn{2}{|c|}{ Prevalence of CLE subtypes among treated subjects: } \\
\hline ACLE: median (range) & $10 \%(0-80 \%)$ \\
\hline SCLE: median (range) & $15 \%(0-85 \%)$ \\
\hline CCLE: median (range) & $50 \%(0-100 \%)$ \\
\hline ICLE: median (range) & $5 \%(0-50 \%)$ \\
\hline
\end{tabular}


Table 2. Usage frequency of different CLE severity measurements in the assessment of treatment efficacy

\begin{tabular}{lccccc}
\hline Variable & PGA & Patient's opinion & CLASI & QoL & SLEDAI \\
\hline USA & $3(88.7 \%)$ & $10(66.7 \%)$ & $4(26.7 \%)$ & $1(6.7 \%)$ & $1(6.7 \%)$ \\
\hline Japan & $27(65.9 \%)$ & $9(22.0 \%)$ & $9(22.0 \%)$ & $8(19.5 \%)$ & $2(4.9 \%)$ \\
\hline Europe & $115(65.2 \%)$ & $11(47.8 \%)$ & $11(47.8 \%)$ & $3(13.0 \%)$ & $1(4.3 \%)$ \\
\hline Other countries & $3(75.0 \%)$ & $3(75.0 \%)$ & $2(50.0 \%)$ & $0(0 \%)$ & $1(25.0 \%)$ \\
\hline$P$-value & 0.46 & $<0.01$ & 0.15 & 0.51 & 0.43 \\
\hline Dermatologists & $55(70.5 \%)$ & $31(39.7 \%)$ & $23(29.5 \%)$ & $10(12.8 \%)$ & $4(5.1 \%)$ \\
\hline Dermatologists and rheumatologists & $2(66.7 \%)$ & $1(33.3 \%)$ & $1(33.3 \%)$ & $2(66.7 \%)$ & $0(100 \%)$ \\
\hline Rheumatologists & $1(50.0 \%)$ & $1(50.0 \%)$ & $2(100 \%)$ & $0(0 \%)$ & $1(50.0 \%)$ \\
\hline$P$-value & 0.82 & 0.93 & 0.1 & 0.03 & 0.03 \\
\hline$<20$ years of professional experience & $26(65.0 \%)$ & $17(42.5 \%)$ & $14(35.0 \%)$ & $5(12.5 \%)$ & $3(7.5 \%)$ \\
\hline$\geq 20$ years of professional experience & $32(74.4 \%)$ & $16(37.2 \%)$ & $12(27.9 \%)$ & $7(16.3 \%)$ & $2(4.7 \%)$ \\
\hline$P$-value & 0.49 & 0.79 & 0.65 & 0.86 & 0.93 \\
\hline$\leq 10$ CLE patients treated per month & $39(72.2 \%)$ & $20(37.0 \%)$ & $15(27.8 \%)$ & $7(13.0 \%)$ & $5(9.3 \%)$ \\
\hline$>10$ CLE patients treater per month & $16(61.5 \%)$ & $12(46.2 \%)$ & $10(38.5 \%)$ & $5(19.2 \%)$ & $0(100 \%)$ \\
\hline$P$-value & 0.48 & 0.59 & 0.48 & 0.69 & 0.27
\end{tabular}

CLASI - Cutaneous Lupus erythematosus Disease Area and Severity Index, CLE - cutaneous lupus erythematosus, PGA - Physician Global Assessment, QOL - quality of life, SLEDAI - Systemic Lupus Erythematosus Disease Activity Index.

SLEDAI ( $n=5,6.0 \%)$ were used much less frequently. One physician (1.2\%) also used photodocumentation to justify the treatment assessment. Physicians from Japan were significantly less willing to make the treatment modification based on the patient's opinion (Table 2). Years of professional experience and number of CLE patients treated per month did not influence the measures used to assess treatment efficacy (Table 2).

A majority of physicians ( $n=32,39.1 \%)$ assessed disease severity at each patient's visit, once a month $(n=$ $4,4.9 \%)$ or at least every third month ( $n=14,17.3 \%)$. Less frequent assessments (every $6^{\text {th }}$ month) were indicated by 5 (6.2\%) participants, and 17 (21.0\%) specialists stated that the frequency of disease severity assessment depended on the treatment chosen for the patients. Interestingly, 2 (2.5\%) physicians did not assess CLE activity at all, and 5 (6.2\%) performed CLE severity measurement irregularly. None of the studied independent variables (country, main working place, specialty, years of professional experience, number of patients seen per month) influenced the frequency of CLE activity assessment (data not shown).

Regarding time needed to consider treatment failure, 39 (47.0\%) physicians waited $2-3$ months before treatment modification. Thirty-one participants indicated that a shorter duration of therapy was needed to define treatment failure: 5 (6.0\%) waited 1 to 2 weeks and 26 (31.3\%) recommended 1 month. Three (3.6\%) physicians suggested longer periods (6 months or longer). Eight (9.6\%) physicians underlined that the decision of treatment failure depended on the chosen treatment modality and one physician also indicated CLE subtype as a factor determining the time needed to evaluate therapy efficacy. One participant did not answer the question regarding time of treatment failure assessment. Interestingly, physicians from Japan waited significantly shorter to consider that the CLE treatment is not effective (1-2 weeks: $5.4 \%$, about 1 month: 59.5\%, 2-3 months: $29.7 \%$, $\geq 6$ months: 5.4\%) than participants from the USA (1-2 weeks: 0\%, about 1 month: 15.3\%, 2-3 months: 76.9\%, $\geq 6$ months: 7.7\%) and Europe (1-2 weeks: $14.3 \%$, about 1 month: 9.5\%, 2-3 months: 76.2\%, $\geq 6$ months: $0 \%)(p<0.01)$.

\section{Photoprotection}

UV exposure is the best documented precipitating or worsening factor of CLE lesions. Therefore, we asked participating physicians about their photoprotection recommendations.

As expected, the vast majority of participating physicians informed their patients about the harmfulness of UV exposure (almost always $n=66,79.5 \%$, in most patients: $n=12,14.5 \%$ ) and most of them recommended some prophylactic measures like sunscreens (almost always: $n=61,73.5 \%$, in most patients: $n=17,20.5 \%$ ) or wearing protective clothes (almost always: $n=55,66.3 \%$, in most patients: $n=18,21.7 \%$ ) (Figures $1-3$ ). Interestingly, physicians from Japan significantly less frequently recommended sunscreens to their patients (sometimes: $12.2 \%$, in most patients: $34.1 \%$, almost always: $53.7 \%$ ) compared to physicians from other countries (USA: 
sometimes: $0 \%$, in most patients: $13.3 \%$, almost always: 86.7\%; Europe: sometimes: 0\%, in most patients: 0\%, almost always: $100 \%$; Other countries: sometimes: $0 \%$, in most patients: $25 \%$, almost always: $75 \%)(p<0.01)$. The Japanese also less often informed their patients about the relationship between UV exposure and CLE lesions, and less frequently recommended other UV prophylactic measures, but the differences did not reach statistical significance (data not shown).

\section{Smoking cessation}

Smoking is another well-documented cause of CLE worsening and treatment resistance. However, in contrast to UV exposure, smoking behavior was less frequently the matter of discussion between physician and CLE patients. Smoking cessation was almost always recommended by about half of participating physicians ( $n=47,56.6 \%)$, whereas 14 (16.9\%) participants based their recommendation to stop smoking on the number of burned cigarettes per day and 9 (10.8\%) on the CLE subtype. Interestingly, $11(13.3 \%)$ rarely and 1 (1.2\%) never mentioned the necessity of smoking cessation to their CLE patients, and one physician did not answer this question, as he indicated that it depends on the treatment type. The only parameter influencing the recommendation for smoking cessation was the country of the participating physicians (Table 3).

\section{Discussion}

A valid and proper assessment tool of CLE severity is still a matter of debate. The CLASI developed by Albrecht and Werth in 2005 is the first instrument designed specifically for evaluating disease activity and skin damage in CLE $[4,10]$. Each of those parameters is helpful in distinct therapeutic areas. The activity score is helpful for short-term drug intervention studies, while the calculation of damage score encourages careful evaluation of lesional activity relative to damage [10]. The damage score may increase as the activity improves. The CLASI has been validated in numerous studies, and was proven to correlate with other clinical outcome instruments, including the physician's global skin assessment, the patient's global skin assessment, the patient's assessment of pain and itch, QoL and a number of biomarker levels $[3,11-17]$. The CLASI is used to assess subtypes of CLE, including acute LE, SCLE, and CCLE, but is not intended for the rarer subsets of CLE, such as lupus panniculitis or bullous LE [18].

The patients' subjective perception of their illness does not always reflect objective disease severity, so assessing QoL in addition to disease severity measures is important [19].

There are also no guidelines regarding the time needed to consider the effectiveness of treatment in CLE, and this needs to be individualized based on the type of

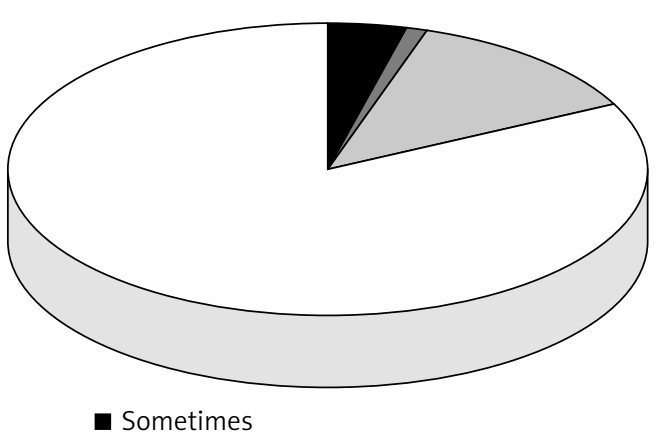

$\square$ Only in patients with known UV hypersensitivity $\square$ In most patients

$\square$ Almost always

Figure 1. How often do you inform your CLE patients about UV effects?

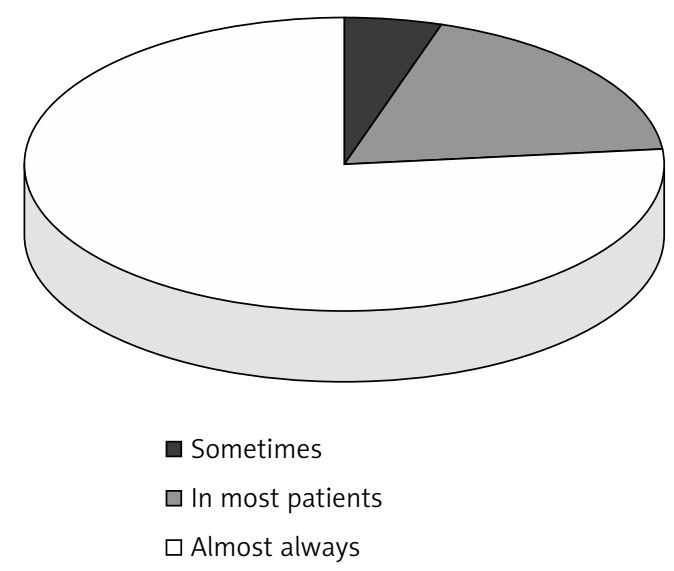

Figure 2. How often do you recommend sunscreens to your CLE patients?

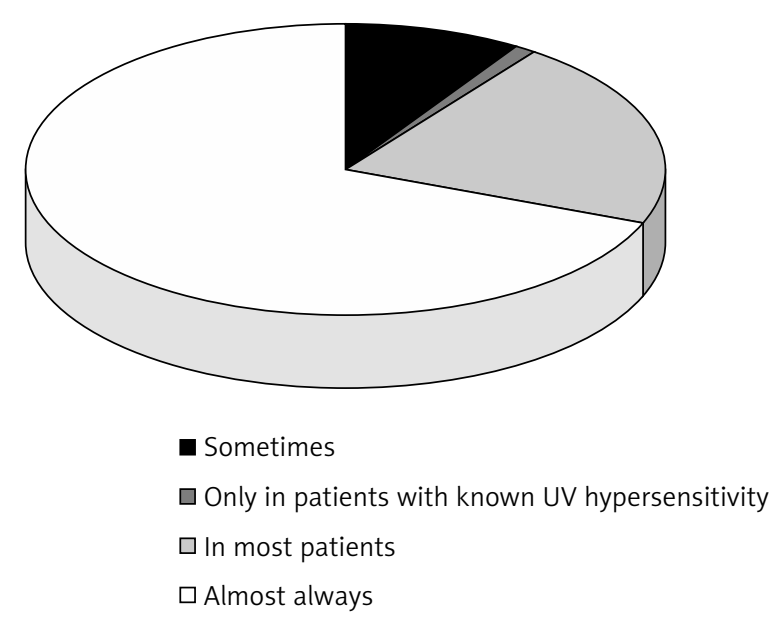

Figure 3. How often do you recommend your patients to use sun protective measures, like wearing long sleeves or having a hat or a cap? 
Table 3. How often do you recommend smoking cessation to your LE patients?

\begin{tabular}{lccccc}
\hline Variable & USA & Japan & Europe & Other countries & $P$-value \\
\hline Almost always & $12(80.0 \%)$ & $11(26.8 \%)$ & $20(87.1 \%)$ & $4(100 \%)$ & 0.001 \\
\hline Sometimes, depending on the LE subtypes & $0(0 \%)$ & $13(31.7 \%)$ & $1(4.3 \%)$ & $0(0 \%)$ & $0(0 \%)$ \\
\hline $\begin{array}{l}\text { Sometimes, depending on the number of } \\
\text { cigarettes per day }\end{array}$ & $1(6.7 \%)$ & $8(19.5 \%)$ & $0(0 \%)$ & $0(0 \%)$ \\
\hline Rarely & $2(13.3 \%)$ & $8(19.5 \%)$ & $1(4.3 \%)$ & $0(0 \%)$ \\
\hline Never & $0(0 \%)$ & $1(2.4 \%)$ & $0(0 \%)$ & $0(0 \%)$ \\
\hline $\begin{array}{l}\text { I cannot answer - depends on treatment } \\
\text { chosen }\end{array}$ & $0(0 \%)$ & $0(0 \%)$ & $1(4.3 \%)$ & & \\
\hline
\end{tabular}

therapy being utilized. Tett et al. have shown that it could take up to 6 months to obtain steady concentration of hydroxychloroquine (HCQ), which further correlates with therapeutic effectiveness of this drug [20]. In addition, clinical studies on SLE patients with skin involvement usually compared the treatment groups 6 months or longer after treatment initiation, further suggesting that the period of 2-3 months could be not long enough to obtain the maximum treatment efficacy [21, 22]. However, there are so many variables that affect the rate of follow up that it could be almost impossible to set up a standardized approach because of the variety of skin lesions, variability of response, severity of disease, and types of therapy used.

Photosensitivity is a frequently observed and welldocumented feature of CLE [23]. Ultraviolet radiation (UVR) is an important exacerbating factor for both cutaneous and systemic symptoms, including lupus nephritis $[24,25]$. Photosensitive rash also correlates with the presence of anti-Ro/SSA, anti-La/SSB and anti-RNP antibodies [26, 27]. The mechanism responsible for induction of CLE-specific skin lesions by UVR is connected with alteration of epidermal antigens and RNA, upregulation of type I interferons, activation of dendritic cells, and lymphocyte recruitment. UVA and UVB are working through different immunological pathways, as UVA penetrates deeper into the dermis, while UVB is primarily responsible for keratinocyte apoptosis and modulation of proinflammatory cytokines, like interleukin-1, tumor necrosis factor alpha and intracellular adhesion molecule-1 [24, 28, 29]. Despite the well-recognized role of UVR in CLE pathogenesis, we have shown that there are some differences regarding recommendations for sun protection depending on the location of participating physicians. Japanese specialists are significantly less likely to recommend sunscreens to their patients relative to physicians from other countries. Such discrepancy may be a result of different habits regarding sun exposure among Asians and Caucasians. Perner et al. [30] showed that, in comparison to Japanese women, German women reported greater exposure to UVR, with more hours of sun exposure and more frequent use of sunbeds. Moreover, more recent studies have shown that the prevalence and the degree of pho- tosensitivity associated with SCLE and lupus erythematosus tumidus is much lower in Japanese patients than in Caucasians [31, 32]. This may influence less frequent recommendations about sun protection among physicians from Japan. In general, it is recommended that all CLE patients apply broad-spectrum sunscreens, as a reaction to UV light might change during the disease [33]. Sunscreen use is a rather cheap and effective prophylactic measure in CLE [34]. Moreover, physicians should educate their patients that lesion formation after UVR exposure may appear up to 3 weeks after sun exposure, making it difficult to accurately assess photosensitivity $[33,35]$. Thus, understanding the correlation between UVR exposure and flares of cutaneous symptoms is crucial for better CLE and SLE disease control. In the authors' opinion, proper education about effective methods of photoprotection should be provided by physicians and other health professionals. This includes daily application of broad-spectrum sunscreens, use of protective clothing and reduction of the overall sun exposure [35].

Another important prophylactic measure in CLE patients is smoking cessation. In recent years the influence of smoking on response to treatment and disease course in CLE has been widely discussed, although some data still remain controversial. However, most experts believe that smoking is a risk factor for refractory CLE and current smokers are less responsive to antimalarial treatment [36]. Recently, Chasset et al. [37] has published a metaanalysis of the literature assessing the interaction between smoking and antimalarial treatment. The results, based on the retrospective analysis of 10 cohort studies, have shown that smoking is associated with a two-fold decrease in the proportion of smokers to non-smokers who had a cutaneous response to antimalarials. Furthermore, smoking was connected with a higher CLE prevalence, and greater cutaneous damage index and scarring [38-40].

\section{Conclusions}

Effective treatment of CLE still remains a challenge. Development of the CLASI has facilitated trials of new 
medications for CLE and skin findings in SLE. Recommendations related to the trial design will be forthcoming in the near future. Finally, physicians need to educate their CLE patients about effective prophylaxis measures related to sunlight and smoking.

\section{Acknowledgments}

We would like to thank all physicians for completing voluntarily the questionnaire: Elizabeth Aberer (Graz, Austria); Peter Heil (Vienna, Austria); Jan Dutz (Vancouver, Canada); Elisabeth O'Brien (Toronto, Canada); Hanna Jedlickova (Brno, Czech Republic); Anette Bygum (Odense, Denmark); Camille Francès (Paris, France); Boutros Soutou (Paris, France); Tsira Leonidze (Tbilisi, Georgia); Regine Gläser (Kiel, Germany); Kerstin Steinbrink (Mainz, Germany); Zsuzsanna Bata-Csörgő (Szeged, Hungary); Emiliano Antiga (Florence, Italy); Marzia Caproni (Florence, Italy); Angelo Valerio Marzano (Milan, Italy); Setsuya Aiba (Miyagi, Japan); Yoshihide Asano (Tokyo, Japan); Ko-Ron Chen (Tokyo, Japan); Manabu Fujimoto (Ibaragi, Japan); Noriki Fujimoto (Shiga, Japan); Etsuko Fujita (Tochigi, Japan); Hiroshi Fujiwara (Niigata, Japan); Toshio Hasegawa (Tokyo, Japan); Hideo Hashizume (Shizuoka; Japan); Hiroaki Hayashi (Okayama, Japan); Hironobu Ihn (Kumamoto, Japan); Hajime lizuka (Hokkaido, Japan); Takaharu Ikeda (Wakayama, Japan); Osamu Ishikawa (Gunma, Japan); Sakae Kaneko (Shimane, Japan); Akira Kasuya (Hamamatsu, Japan); Seiji Kawana (Tokyo, Japan); Nobuhiko Kobayashi (Nara, Japan); Masaya Kodera (Aichi, Japan); Hiroshi Koga (Nagano, Japan); Yutaka Kuwatsuka (Nagasaki, Japan); Teruhiko Makino (Toyama, Japan); Yasushi Matsuzaki (Aomori, Japan); Hitoshi Mizutani (Mie, Japan); Yoko Momose (Tokyo, Japan); Shinichi Moriwaki (Osaka, Japan); Yoshinao Muro (Aichi, Japan); Motonobu Nakamura (Fukuoka, Japan); Ken Natsuga (Hokkaido, Japan); David Norris (Denver, CO, USA); Toshio Ootani (Okayama, Japan); Shigetoshi Sano (Kochi, Japan); Tetsuo Shiohara (Tokyo, Japan); Yoshinao Soma (Kanagawa, Japan); Tamio Suzuki (Yamagata, Japan); Takao Tachibana (Osaka, Japan); Kazuo Takahashi (Kanagawa, Japan); Kouichirou Takeda (Kagoshima, Japan); Akiko Tanikawa (Tokyo, Japan); Tetsuya Tsuchida (Saitama, Japan); Ikuko Ueda (Osaka, Japan); Toshiyuki Yamamoto (Fukushima, Japan); Naoto Yokogawa (Tokyo, Japan); Rafał BiałynickiBirula (Wrocław, Poland); Ewa Niezgoda (Żary, Poland); Tiago Torres (Porto, Portugal); Sue Jessop (Cape Town, Republic of South Africa); Rodica Olteanu (Bucharest, Romania); Dusan Skiljevic (Belgrade, Serbia); Pilar Iranzo (Barcelona, Spain); Júlia María Sánchez Schmidt (Barcelona, Spain); Carlo Mainetti (Bellinzona, Switzerland); Jeffrey Callen (Louisville, KY, USA); Benjamin Chong (Dallas, TX, USA); Jennie Clarke (Hershey, PA, USA); Justin Endo (Madison, WI, USA); Nicole Fett (Portland, OR, USA); David Fiorentino (Redwood City, CA, USA); J. Mark Jackson (Louisville, KY, USA); Anne Laumann (Chicago, IL, USA);
Lela Lee (Denver, CO, USA); Jami L Miller (Nashville, TN, USA); Sabrina Newman (Washington, DC, USA); Alex Ortega-Loayza (Richmond, VA, USA); Richard Sontheimer (Worcester, MA, USA).

\section{Conflict of interest}

The authors declare no conflict of interest.

\section{References}

1. Kuhn A, Ruzicka T. Classification of cutaneous lupus erythematosus. In: Cutaneous Lupus Erythematosus. Kuhn A, Lehmann P, Ruzicka T (eds). Springer-Verlag, Berlin 2004; 53-8.

2. Hejazi EZ, Werth VP. Cutaneous lupus erythematosus: an update on pathogenesis, diagnosis and treatment. Am J Clin Dermatol 2016; 17: 135-46.

3. Klein RS, Morganroth PA, Werth VP. Cutaneous lupus and the CLASI Instrument. Rheum Dis Clin North Am 2010; 36: 33-51.

4. Kuhn A, Meuth AM, Bein D, et al. Revised Cutaneous Lupus Erythematosus Disease Area and Severity Index (RCLASI): a modified outcome instrument for cutaneous lupus erythematosus. Br J Dermatol 2010; 163: 83-92.

5. Albrecht J, Taylor L, Berlin JA, et al. The CLASI (Cutaneous Lupus Erythematosus Disease Area and Severity Index): an outcome instrument for cutaneous lupus erythematosus. J Invest Dermatol 2005; 125: 889-94.

6. Khamashta M, Merrill JT, Werth VP, et al. Sifalimumab, an anti-interferon-alpha monoclonal antibody, in moderate to severe systemic lupus erythematosus: a randomised, double-blind, placebo-controlled study. Ann Rheum Dis 2016; 75 : 1909-16.

7. Furie R, Khamashta M, Merrill JT, et al. Anifrolumab, an antiinterferon-alpha receptor monoclonal antibody, in moderate to severe systemic lupus erythematosus. Arthritis Rheumatol 2017; 69: 376-86.

8. Vasquez R, Tseng LC, Victor S, Zhang S, Chong BF. Autoantibody and clinical profiles in patients with discoid lupus and borderline systemic lupus. Arch Dermatol 2012; 148: 651-5.

9. Reich A, Werth VP, Furukawa F, et al. Treatment of cutaneous lupus erythematosus: current practice variations. Lupus 2016; 25: 964-72.

10. Albrecht J, Werth VP. Development of the CLASI as an outcome instrument for cutaneous lupus erythematosus. Dermatol Ther 2007; 20: 93-101.

11. Bonilla-Martinez Z, Albrecht J, Taylor L, et al. The Cutaneous Lupus Erythematosus Disease Area and Severity Index: a responsive instrument to measure activity and damage in patients with cutaneous lupus erythematosus. Arch Dermatol 2008; 144: 173-80.

12. Chang AY, Ghazi E, Okawa J, et al. Quality of life differences between responders and non-responders in the treatment of cutaneous lupus erythematosus. JAMA Dermatol 2013; 149: 104-6.

13. Klein R, Moghadam-Kia S, LoMonico J, et al. Development of the CLASI as a tool to measure disease severity and responsiveness to therapy in cutaneous lupus erythematosus. Arch Dermatol 2011; 147: 203-8.

14. Vasquez R, Wang D, Tran QP, et al. A multi-center, crosssectional study on quality of life in cutaneous lupus erythematosus. Br J Dermatol 2012; 168: 145-53. 
15. Braunstein I, Klein R, Okawa J, et al. The interferon-regulated gene signature is elevated in subacute cutaneous lupus erythematosus and discoid lupus erythematosus and correlates with the cutaneous lupus area and severity index score. Br J Dermatol 2012; 166: 971-5.

16. Okon LG, Werth VP. Cutaneous lupus erythematosus: diagnosis and treatment. Best Pract Res Clin Rheumatol 2013; 27: 391-404.

17. Dutz J, Werth VP. Cigarette smoking and response to antimalarials in CLE patients: evolution of a dogma. J Invest Dermatol 2011; 131: 1968-70.

18. Avilés Izquierdo JA, Cano Martinez N, Lázaro Ochaita P. Epidemiological characteristics of patients with cutaneous lupus erythematosus. Actas Dermosifiliogr 2014; 105: 69-73.

19. Klein R, Moghadam-Kia S, Taylor L, et al. Quality of life in cutaneous lupus erythematosus. I Am Acad Dermatol 2011; 64: 849-58.

20. Tett SE, Day RO, Cutler DJ. Concentration-effect relationship of hydroxychloroquine in rheumatoid arthritis - a cross sectional study. J Rheumatol 1993; 20: 1874-9.

21. Bezerra EL, Vilar MJ, da Trindade Neto PB, et al. Double-blind, randomized, controlled clinical trial of clofazimine compared with chloroquine in patients with systemic lupus erythematosus. Arthritis Rheum 2005; 52: 3073-8.

22. Gansauge S, Breitbart A, Rinaldi N, et al. Methotrexate in patients with moderate systemic lupus erythematosus (exclusion of renal and central nervous system disease). Ann Rheum Dis 1997; 56: 382-5.

23. Kuhn A, Beissert S. Photosensitivity in lupus erythematosus. Autoimmunity 2005; 38: 519-29.

24. Zandman-Goddard G, Solomon M, Rosman Z, et al. Environment and lupus related diseases. Lupus 2012; 21: 241-50.

25. Schmidt E, Tony HP, Brocker EB, et al. Sun-induced lifethreating lupus nephritis. Ann N Y Acad Sci 2007; 1108: 35-40.

26. Mond CB, Peterson MG, Rothfield NF. Correlation of anti-Ro antibody with photosensitivity rash in systemic lupus erythematosus patients. Arthritis Rheum 1989; 32: 202-4.

27. Wolska H, Blaszczyk M, Jablonska S. Phototest in patients with various forms of lupus erythematosus. Int I Dermatol 1989; 28: 98-103.

28. Furukawa $\mathrm{F}$, Itoh $\mathrm{T}$, Wakita $\mathrm{H}$, et al. Keratinocytes from patients with lupus erythematosus show enhanced cytotoxicity to ultraviolet radiation and to antibody-mediated cytotoxicity. Clin Exp Immunol 1999; 118: 164-70.

29. Köck A, Schwarz T, Kirnbauer R, et al. Human keratinocytes are a source for tumor necrosis factor alpha: evidence for synthesis and release upon stimulation with endotoxin or ultraviolet light. J Exp Med 1990; 172: 1609-14.

30. Perner D, Vierkötter A, Sugiri D, et al. Association between sun-exposure, smoking behaviour and plasma antioxidant levels with the different manifestation of skin ageing signs between Japanese and German women - a pilot study. J Dermatol Sci 2011; 62: 138-40.

31. Furukawa F, Muto M. Ethnic differences in immunogenetic features and photosensitivity of cutaneous lupus erythematosus. Arch Dermatol Res 2009; 301: 111-5.

32. Furukawa F, Yamamoto $Y$, Kanazawa N, et al. Race differences in immunogenetic features and photosensitivity of $\mathrm{Cu}$ taneous lupus erythematosus from the aspect of Japanese studies. Ann N Y Acad Sci 2009; 1173: 552-6.

33. Ruland V, Haust M, Stilling RM, et al. Updated analysis of standardized photoprovocation in patients with cutaneous lupus erythematosus. Arthritis Care Res 2013; 65: 767-76.
34. Kuhn A, Gensch K, Haust M, et al. Photoprotective effects of a broad-spectrum sunscreen in ultraviolet-induced cutaneous lupus erythematosus: a randomized, vehicle-controlled, double-blind study. J Am Acad Dermatol 2011; 64: 37-48.

35. Kuhn A, Ruland V, Bonsmann G. Photosensitivity, phototesting, and photoprotection in cutaneous lupus erythematosus. Lupus 2010; 19: 1036-46.

36. Leroux G, Costedoat-Chalumenasu N, Hulot JS. Relationship between blood hydroxychloroquine and desenthylchloroquine concentration and cigarette smoking in treated patients with connective tissue diseases. Ann Rheum Dis 2007; 66: 1547-8.

37. Chasset F, Francès $C$, Barete $S$. Influence of smoking on the efficacy of antimalarials in cutaneous lupus erythematosus: a meta-analysis of the literature. J Am Acad Dermatol 2015; 72: 634-9.

38. Ezra N, Jorizzo J. Hydroxychloroquine and smoking in patients with cutaneous lupus erythematosus. Clin Exp Dermatol 2012; 37: 327-34

39. Piette EW, Foering KP, Chang AY, et al. Impact of smoking in cutaneous lupus erythematosus. Arch Dermatol 2012; 148: 317-22.

40. Turchin I, Bernatsky S, Clarke AE, et al. Cigarette smoking and cutaneous damage in systemic lupus erythematosus. J Rheumatol 2009; 36: 2691-3. 